Pacific Journal of Mathematic 


\title{
$K$-THEORY FOR COMMUTANTS IN THE CALKIN ALGEBRA
}

\author{
William L. PASChKe
}

\begin{abstract}
Let $A$ be a separable unital $C^{*}$-algebra, and let $B^{c}$ be the commutant in the Calkin algebra of the image $B$ of $A$ under a trivial extension. We show that $K_{0}\left(B^{c}\right)$ is isomorphic to the group of invertibles in (weak) Ext of $A$ and that, in the presence of an appropriate homotopy invariance assumption, $K_{1}\left(B^{c}\right)$ is isomorphic to Ext of the reduced suspension of $A$. These facts lead to an alternative approach to the Pimsner-Voiculescu exact sequence for Ext of a crossed product.
\end{abstract}

This paper originated from the following simple observation. Let $\theta$ be a ${ }^{*}$-automorphism of $A$, and suppose that the crossed product of $A$ by $\theta$ is faithfully represented on (separable, infinite-dimensional) Hilbert space $H$ as $C^{*}\left(A, U_{\theta}\right)$, the $C^{*}$-algebra generated by $A$ and $\theta$-implementing unitary $U_{\theta}$, with $C^{*}\left(A, U_{\theta}\right) \cap$ (compacts) $=0$. Let $\pi: L(H) \rightarrow Q(H)$ (Calkin algebra) be the Calkin map. Then any unitary $w$ in $\pi(A)^{c}$ (the commutant of $\pi(A)$ in $Q(H)$ ) gives rise to a *-homomorphism $\tau: C^{*}\left(A, U_{\theta}\right) \rightarrow Q(H)$ taking $x$ in $A$ to $\pi(x)$ and $U_{\theta}$ to $\pi\left(U_{\theta}\right) w$. (This is because conjugation by $\pi\left(U_{\theta}\right) w$ has the same effect on $\pi(A)$ as conjugation by $\pi\left(U_{\theta}\right)$.) Direct-summing with a trivial extension gives an extension of $C^{*}\left(A, U_{\theta}\right)$ corresponding to $w$. When $C^{*}\left(A, U_{\theta}\right)$ has the homotopy-invariance property (extensions joinable by a path are equivalent), two unitaries which can be joined by a path of unitaries commuting with $\pi(A)$ give rise to equivalent extensions.

The correspondence we have indicated here comes up (at least implicitly) in the computation by Popa and Rieffel [9] of Ext of the irrational rotation algebras. We will see below that it induces a map from $K_{1}\left(\pi(A)^{c}\right)$ to $\operatorname{Ext}\left(C^{*}(A, \theta)\right)$ which, when properly decoded, is one of the boundary maps in the Pimsner-Voiculescu exact sequence [6].

The only deep results that we will need are 1.5 and 1.6 of Voiculescu's seminal paper [12] (see also [1]). In the language of extensions, these say that any two trivial extensions (extensions factorable through representations on $H$ ) are equivalent (conjugate by an index-zero unitary in $Q(H)$ ), and that the direct sum of any extension with a trival extension is equivalent to the given extension. We will refer to these two facts together as "Voiculescu's theorem" and make frequent use of them, generally without comment. All we will use from $K$-theory are the definitions of $K_{0}$ (resp. $\left.K_{1}\right)$, for which 
we provide a reference (resp. reminder) at the appropriate moment. Our main project is to identify $K_{1}\left(\pi(A)^{c}\right)$ in extension-theoretic terms, but it will be helpful to consider $K_{0}$ first. We assume throughout that $A$ is faithfully represented on $H$ with $A \cap$ (compacts) $=$ (0). The commutant of the image of $A$ in $Q(H)$ under any trivial extension is isomorphic to $\pi(A)^{c}$ by Voiculescu's theorem. In particular, if we write $H^{n}$ for the $n$-fold direct sum of $H$ with itself and $\pi^{n}: L(H) \rightarrow Q\left(H^{n}\right)$ for the map that takes $T$ in $L(H)$ to the image in $Q\left(H^{n}\right)$ of the $n$-fold direct sum of $T$ with itself, then $\pi^{n}(A)^{c}$ and $\pi(A)^{c}$ are isomorphic. Notice also that $\pi^{n}(A)^{c}$ may be identified with $\pi(A)^{c} \otimes M_{n}$, the algebra of $n \times n$ matrices over $\pi(A)^{\circ}$. For the definition of $K_{0}$ of a $C^{*}$-algebra we refer the reader to $\S 3$ of [4]. Throughout this paper, $\operatorname{Ext}(A)$ will mean weak Ext, the semigroup of weak equivalence classes of extensions of $A$. We write $\operatorname{Ext}^{*}(A)$ for the group of invertible elements of $\operatorname{Ext}(A)$.

LEMMA 1. The equivalence classes in $K_{0}\left(\pi(A)^{\circ}\right)$ of projections in $\pi(A)^{c}$ constitute all of $K_{0}\left(\pi(A)^{c}\right)$.

Proof. Let $p$ be a projection in $\pi(A)^{c}$ and let $\sigma_{0}: C^{*}(\pi(A), p) \rightarrow$ $Q(H)$ be a trivial extension. By Voiculescu's theorem we may assume that $\sigma_{0}(\pi(x))=\pi(x)$ for $x$ in $A$; furthermore, $\sigma_{0}$ is equivalent to $\sigma_{0} \oplus$ $\sigma_{0}$, so there is a $v$ in $Q\left(H^{2}\right)$ such that $v\left(\pi^{2}(x)\right) v^{*}=\pi(x) \oplus 0(x$ in $A)$, $v\left(\sigma_{0}(p) \oplus \sigma_{0}(p)\right) v^{*}=\sigma_{0}(p) \oplus 0, v^{*} v=1 \oplus 1$, and $v v^{*}=1 \oplus 0$. We have $v \pi^{2}(x)=\pi^{2}(x)(1 \oplus 0) v=\pi^{2}(x) v$ for $x$ in $A$, so $\left[\sigma_{0}(p)\right]_{0}+\left[\sigma_{0}(p)\right]_{0}=\left[\sigma_{0}(p)\right]_{0}$, where [ $]_{0}$ denotes the equivalence class in $K_{0}\left(\pi(A)^{c}\right)$. Hence $\left[\sigma_{0}(p)\right]_{0}=$ 0. Applying this to $1_{n}=\pi^{n}(1)$ in $\pi^{n}(A)$, we have $\left[1_{n}\right]_{0}=0$ and so for all projections $q$ in $\pi(A)^{c} \otimes M_{n}$ (which we identify with $\pi^{n}(A)^{c}$ ), $\left[1_{n}-q\right]_{0}=-[q]_{0}$. That is, equivalence classes of projections in $\pi^{n}(A)^{\circ}(n \geqq 1)$ form a group. Now take $n>1$ and let $q$ be a projection in $\pi^{n}(A)^{c}$. Let $\sigma: C^{*}\left(\pi^{n}(A), q\right) \rightarrow Q(H)$ be an extension equivalent to the inclusion of this algebra in $Q\left(H^{n}\right)$, and let $\sigma_{0}: C^{*}\left(\pi^{n}(A), q\right) \rightarrow$ $Q\left(H^{n-1}\right)$ be a trivial extension. We may assume that $\sigma\left(\pi^{n}(x)\right)=\pi(x)$ and $\sigma_{0}\left(\pi^{n}(x)\right)=\pi^{n-1}(x)$ for $x$ in $A$. The equivalence of $\sigma \oplus \sigma_{0}$ with the inclusion map yields a unitary $w$ in $\pi^{n}(A)^{c}$ such that $w q w^{*}=$ $\sigma(q) \oplus \sigma_{0}(q)$, so $[q]_{0}=[\sigma(q)]_{0}+\left[\sigma_{0}(q)\right]_{0}=[\sigma(q)]_{0}$. This proves the lemma.

TheOREM 2. Let $A$ be a separable unital $C^{*}$-algebra. Then $K_{0}\left(\pi(A)^{c}\right)$ is isomorphic to $\operatorname{Ext}^{*}(A)$.

Proof. The idea here is simply that invertible extensions of $A$ are cutdowns of $\left.\pi\right|_{A}$ by projections in $\pi(A)^{c}$. Let $p$ be such a projection. Direct-summing $p$ with 1 (and pulling back to $\left.\pi(A)^{c}\right)$, we may assume that $x \rightarrow \pi(x) p$ is a ${ }^{*}$-monomorphism. Let $v$ in $Q(H)$ be 
such that $v^{*} v=1$ and $v v^{*}=p$. Then $\tau: A \rightarrow Q(H)$ defined by $\tau(x)=v^{*} \pi(x) v$ is an extension of $A$. If $p_{1}$ is another projection in $\pi(A)^{c}$ with $[p]_{0}=\left[p_{1}\right]_{0}$, then (direct-summing with $1_{n}$ if necessary and pulling back to $\left.\pi(A)^{c}\right)$ we may assume that there is a $w$ in $\pi(A)^{c}$ with $w^{*} w=p, w w^{*}=p_{1}$. Let $\tau_{1}(x)=v_{1}^{*} \pi(x) v_{1}$, where $v_{1}^{*} v_{1}=1$ and $v_{1} v_{1}^{*}=p_{1}$. One checks that $v^{*} w^{*} v_{1}$ is unitary and implements an equivalence between $\tau_{1}$ and $\tau$. Thus, the correspondence $p \rightarrow \tau$ gives rise to a map $\beta: K_{0}\left(\pi(A)^{c}\right) \rightarrow \operatorname{Ext}(A)$ with $\beta\left([p]_{0}\right)=[\tau]$. It is clear that $\beta$ is additive and takes 0 to 0 , and hence $\beta$ maps into the group of invertibles in $\operatorname{Ext}(A)$. It is also clear that $\beta$ maps onto the group of invertibles. Finally, if $\beta\left([p]_{0}\right)=0$, then with $\tau$ and $v$ as above, there exists a unitary $u$ in $Q(H)$ such that $u \tau(x) u^{*}=$ $u v \pi(x) v^{*} u^{*}=\pi(x)$ for $x$ in $A$, so $[p]_{0}=[1]_{0}=0$ via $u v$ in $\pi(A)^{c}$.

We turn to the identification of $K_{1}\left(\pi(A)^{c}\right)$. Write $U\left(\pi(A)^{c}\right)$ for the unitary group of $\pi(A)^{c}$ and let $I\left(\pi(A)^{c}\right)$ be the quotient of $U\left(\pi(A)^{c}\right)$ by the path-connected component of 1 , with $\langle\cdot\rangle: U\left(\pi(A)^{c}\right) \rightarrow I\left(\pi(A)^{c}\right)$ the quotient map. Most of the bookkeeping necessary for what follows is taken care of in the following lemma. (The proof uses much the same techniques as those in [9].)

Lemma 3. Let $A$ be as above.

(1) For $u$ in $U\left(\pi(A)^{c}\right)$, let $\sigma_{0}: C^{*}(\pi(A), u) \rightarrow Q(H)$ be a trivial extension such that $\sigma_{0}(\pi(x))=\pi(x)$ for $x$ in $A$. Then $\left\langle\sigma_{0}(u)\right\rangle=\langle 1\rangle$.

(2) The map $\langle u\rangle \rightarrow\langle u \oplus 1\rangle$ from $I\left(\pi(A)^{c}\right)$ to $I\left(\pi^{2}(A)^{c}\right)$ is in jective.

(3) $I\left(\pi(A)^{c}\right)$ is abelian.

(4) The map in (2) is surjective, hence an isomorphism.

Proof. (1) If $\rho: C^{*}(\pi(A), u) \rightarrow L(H)$ is a lifting for $\sigma_{0}$, then $\rho\left(\sigma_{0}(u)\right)$ belongs to the von Neumann algebra $\rho(\pi(A))^{\prime}$ and hence has a logarithm commuting with $\rho(\pi(A))$. This enables us to join $\rho\left(\sigma_{0}(u)\right)$ to 1 by a path of unitary operators commuting with $\rho(\pi(A))$. The image of this path in the Calkin algebra joins $\sigma_{0}(u)$ to 1 by a path in $U\left(\pi(A)^{c}\right)$.

(2) Let $u$ in $U\left(\pi(A)^{c}\right)$ be such that $\langle u \oplus 1\rangle=\langle 1 \oplus 1\rangle$ in $I\left(\pi^{2}(A)^{c}\right)$, and let $\sigma_{0}: C^{*}(\pi(A), u) \rightarrow Q(H)$ be a trivial extension. We may assume that $\sigma_{0}(\pi(x))=\pi(x)$ for $x$ in $A$, so if we set $v=\sigma_{0}(u)$, we have $\langle v\rangle=$ $\langle 1\rangle$ by part (1). Moreover, the extension $i \oplus \sigma_{0}: C^{*}(\pi(A), u) \rightarrow Q(H)^{2}$, where $i$ is the inclusion map, is strongly equivalent to $i$, so there is a ${ }^{*}$-isomorphism $\varphi: Q\left(H^{2}\right) \rightarrow Q(H)$ (induced by a unitary map of $H^{2}$ onto $H$ ) satisfying $\varphi\left(\pi^{2}(x)\right)=\pi(x)$ for $x$ in $A$ and $\varphi(u \oplus v)=u$. Now $\langle u \oplus v\rangle=\langle u \oplus 1\rangle=\langle 1 \oplus 1\rangle$, that is, there is a path in $U\left(\pi^{2}(A)^{c}\right)$ joining $u \oplus v$ to $1 \oplus 1$. The image of this path under $\varphi$ joins $u$ to 1 in $U\left(\pi(A)^{c}\right)$.

(3) This follows from part (2) by a standard argument. If $u_{1}, u_{2} \in U\left(\pi(A)^{c}\right)$ and $S$ is the $2 \times 2$ scalar matrix interchanging the 
standard basis vectors, then $\left\langle u_{1} u_{2} \oplus 1\right\rangle=\left\langle\left(u_{1} \oplus 1\right)\left(u_{2} \oplus 1\right)\right\rangle=$ $\left\langle\left(u_{1} \oplus 1\right) S\left(u_{2} \oplus 1\right) S\right\rangle=\left\langle u_{1} \oplus u_{2}\right\rangle=\left\langle u_{2} \oplus u_{1}\right\rangle=\left\langle u_{2} u_{1} \oplus 1\right\rangle$ because $S$ can be joined to $1 \oplus 1$ by a path of scalar matrices. By part (2), then, $\left\langle u_{1} u_{2}\right\rangle=\left\langle u_{2} u_{1}\right\rangle$.

(4) Take $w$ in $U\left(\pi^{2}(A)^{c}\right)$. We must produce a $u$ in $U\left(\pi(A)^{c}\right)$ such that $\langle u \oplus 1\rangle=\langle w\rangle$. Let $\tau_{0}$ and $\tau: C^{*}\left(\pi^{2}(A), w\right) \rightarrow Q(H)$ be, respectively, a trivial extension and an extension equivalent to the inclusion $i$ of this algebra in $Q\left(H^{2}\right)$. Since both $\tau_{0}$ and $\tau$ have trivial restrictions to $\pi^{2}(A)$, we may assume that they both send $\pi^{2}(x)$ to $\pi(x)$ for $x$ in $A$. Set $u_{0}=\tau_{0}(w), u=\tau(w)$. Now $\tau \oplus \tau_{0}$ is equivalent to $i$, so there is a unitary $v$ in $Q\left(H^{2}\right)$ such that $v b v^{*}=\left(\tau \oplus \tau_{0}\right)(b)$ for all $b$ in $C^{*}\left(\pi^{2}(A), w\right)$. In particular, $v$ commutes with $\pi^{2}(A)$, so by part (3), $\left\langle v w v^{*}\right\rangle=\langle w\rangle$. But $\left\langle v w v^{*}\right\rangle=\left\langle u \oplus u_{0}\right\rangle$, and $\left\langle u_{0}\right\rangle=\langle 1\rangle$ by part (1), so $\langle w\rangle=\langle u \oplus 1\rangle$ as required.

Proposition 4. With $A$ as above, the groups $K_{1}\left(\pi(A)^{c}\right)$ and $I\left(\pi(A)^{c}\right)$ are isomorphic.

Proof. Every invertible $b$ in a unital $C^{*}$-algebra $B$ can be joined by a path of invertibles to its "unitary part" $b|b|^{-1}$, and two invertibles can be joined by a path of invertibles if and only if their unitary parts can be joined by a path of unitaries. The definition in [11] thus gives $K_{1}(B)$ as the inductive limit of the system

$$
\psi_{n, n+k}: I\left(B \otimes M_{n}\right) \longrightarrow I\left(B \otimes M_{n+k}\right),
$$

where $\psi_{n, n+k}(\langle u\rangle)=\left\langle u \oplus 1_{k}\right\rangle$. In our situation, $\pi(A)^{c} \otimes M_{n}$ is isomorphic to $\pi^{n}(A)^{c}$, which in turn is isomorphic to $\pi(A)^{c}$. By the previous lemma, all of the connecting maps in the system are isomorphisms, so taking the inductive limit simply produces $I\left(\pi(A)^{c}\right)$.

In light of Proposition 4, we will use $\langle w\rangle$ to denote the element of $K_{1}\left(\pi(A)^{c}\right)$ represented by $w$ in $U\left(\pi(A)^{c}\right)$.

Our next result in a straightforward consequence of Lemma 3 that may be of some general interest. Here, $T$ is the unit circle.

Proposition 5. Let $A$ be as above and suppose that the map from $\operatorname{Ext}(A \otimes C(T))$ to $\operatorname{Ext}(A) \oplus Z$ induced by restriction to $A \otimes 1$ and $1 \otimes C(T)$ is injective. Then any $w$ in $U\left(\pi(A)^{c}\right)$ of index 0 can be joined to 1 by a path in $U\left(\pi(A)^{c}\right)$.

Proof. Let $A \otimes C(T)$ be faithfully represented, missing the compacts, on $H$ and let $U$ in $A^{\prime}$ be unitary with $A \otimes C(T)=C^{*}(A, U)$. By the universal property of the tensor product, there is an extension $\tau: C^{*}(A, U) \rightarrow Q\left(H^{2}\right)$ such that $\tau(x)=\pi^{2}(x)(x$ in $A)$ and $\tau(U)=w \oplus$ 
$\pi(U)$. Because of our assumption on $\operatorname{Ext}(A \otimes C(T)), \tau$ is trivial. There is thus a unitary $v$ in $\pi(A)^{c}$ such that $v(w \oplus \pi(U)) v^{*}=\pi^{2}(U)$. By parts (1) and (3) of Lemma 3, we have $\langle w \oplus 1\rangle=\langle w \oplus \pi(U)\rangle=$ $\left\langle v(w \oplus \pi(U)) v^{*}\right\rangle=\langle 1 \oplus 1\rangle$, so $\langle w\rangle=\langle 1\rangle$ by part (2).

It follows from Theorem 7 below (see also [3] and 2.4 of [2]) that $A=C(T)$ satisfies the condition of the proposition, so if $u$ and $v$ are commuting index-zero unitaries in $Q(H)$ and the spectrum of $u$ is $T$, then $v$ can be joined to 1 by a path of unitaries commuting with $u$. (This is also shown as part of the proof of the main result in [9].)

Following the usage in [6] (as opposed to [8]), we say that $A$ has the homotopy-invariance property if whenever two extensions $\tau_{0}, \tau_{1}: A \rightarrow Q(H)$ can be joined by a (point-norm continuous) path of extensions, we have $\tau_{0}$ strongly equivalent to $\tau_{1}$. In [8], g.q.d. algebras, among which are the quasidiagonal algebras of [10], were defined and shown to have the homotopy-invariance property. There are many g.q.d. algebras. Moreover, it is possible to establish homotopy invariance for certain non-g.q.d. algebras on an ad hoc basis; for instance, the computations in [5] or [7] show fairly readily that the Cuntz algebras $O_{n}$ have this property. In the theorem below, $\Omega A$ is the reduced suspension of $A$, that is, the $C^{*}$-algebra of continuous functions from $T$ to $A$ which are scalar-valued at 1.

THEOREM 6. Let $A$ be a separable unital $C^{*}$-algebra and suppose that $A \otimes C(T)$ has the homotopy-invariance property. Then $K_{1}\left(\pi(A)^{c}\right)$ is isomorphic to $\operatorname{Ext}^{*}(\Omega A)$.

Proof. With the same set-up as in the proof of Proposition 5, $\Omega A$ may be identified with a subalgebra of $C^{*}(A, U)$, namely the subalgebra generated by 1 and $\{x(f(U)-f(1)): x \in A, f \in C(T)\}$. (Notice that $U$ belongs to $\Omega A$.) We will exhibit an isomorphism between $K_{0}\left(\pi(\Omega A)^{c}\right)$ and $K_{1}\left(\pi(A)^{c}\right)$, using Theorem 2 to identify the former with $\operatorname{Ext}^{*}(\Omega A)$. Define $\gamma: K_{0}\left(\pi(\Omega A)^{c}\right) \rightarrow K_{1}\left(\pi(A)^{c}\right)$ by $\gamma\left([p]_{0}\right)=\langle w\rangle$, where $p$ is a projection in $\pi(\Omega A)^{c}$ and $w=p \pi(U)+1-p$. (Since $p$ commutes with $\pi(U), w$ is unitary. For $x$ in $A, p$ commutes with $\pi(x)(\pi(U)-1)$, so $p(\pi(U)-1)$ commutes with $\pi(A)$, so $w=$ $p(\pi(U)-1)+1$ commutes with $\pi(A)$.) In order to check that $\gamma$ is well-defined, let $q$ be another projection in $\pi(\Omega A)^{c}$, giving rise to a unitary $v$ in $\pi(A)^{c}$ as above, with $[q]_{0}=[p]_{0}$. Since $\langle w\rangle$ is unchanged by direct-summing $\pi(U)$ with $1_{n}$, and $p$ with $1_{n}$ or $0_{n}$, and then pulling back to $\pi(A)^{c}$ (use Lemma 3 to see this), we may assume that $p q=0$ and that there is a partial isometry $s$ in $\pi(\Omega A)^{c}$ such that $s^{*} s=p, s s^{*}=q$. Notice that $\left\{p, s, s^{*}, q\right\}$ is a system of $2 \times 2$ matrix units in $\pi(\Omega A)^{c}$, so $p$ and $q$ can be joined by a path of 
projections in $\pi(\Omega A)^{c}$, whence it follows that $\langle w\rangle=\langle v\rangle$. Since $\left\langle w_{1} \oplus w_{2}\right\rangle=\left\langle w_{1} w_{2}\right\rangle$ in $K_{1}\left(\pi(A)^{c}\right)$ (see the proof of part (3) of Lemma 3 ), the map $\gamma$ is a homomorphism. We now show that $\gamma$ is injective. Let $p$ and $w$ be as above and suppose that $\langle w\rangle=\langle 1\rangle$. Directsumming $p$ with 1 and $w$ with $\pi(U)$ if necessary, $\left([p]_{0}=[p \oplus 1]_{0}\right.$ by the proof of Theorem $2,\langle\pi(U)\rangle=\langle 1\rangle$ by Lemma 3), we use the universal property of the tensor product to obtain an extension $\tau: C^{*}(A, U) \rightarrow Q(H)$ with $\tau(x)=\pi(x)$ for $x$ in $A$ and $\tau(U)=w$. Since $\langle w\rangle=\langle 1\rangle$, the direct sum of $\tau$ with a trivial extension can be joined by a path to a trivial extension. Thus the assumed homotopy invariance of $C^{*}(A, U)$ implies that $\tau$ is trivial. Let $v$ in $U\left(\pi(A)^{\circ}\right)$ be such that $v w v^{*}=v p \pi(U) v^{*}+v(1-p) v^{*}=\pi(U)$. Multiplying by $v p$ on the right shows that $v p$ commutes with $\pi(U)$. In fact, $v p \in$ $\pi(\Omega A)^{c}$ because if $f \in C(T)$ with $f(1)=0$ and $x \in A$, then

$$
v p \pi(x) \pi(f(U))=v \pi(x) \pi(f(U)) p=(\pi(x) v)(p \pi(f(U)))=\pi(x) \pi(f(U)) v p .
$$

Thus $[p]_{0}=\left[v p v^{*}\right]_{0}$ in $K_{0}\left(\pi(\Omega A)^{c}\right)$ and we may assume that $\pi(U) p+$ $1-p=\pi(U)$. Set $q=1-p$, so $\pi(U) q=q$. It follows that $\pi(y) q=$ $\phi(y) q$ for $y \in \Omega A$, where $\phi: \Omega A \rightarrow C$ is the (unique) character with $\phi(U)=1$. The extension of $\Omega A$ to which $q$ corresponds is therefore trivial and we have $0=[q]_{0}=[1-p]_{0}=-[p]_{0}$, so $\gamma$ is injective. To check surjectivity, finally, take $w$ in $U\left(\pi(A)^{c}\right)$. Direct-summing $w$ with $\pi(U)$ if necessary, we may assume that there is an extension $\tau: C^{*}(A, U) \rightarrow Q(H)$ with $\tau(x)=\pi(x)(x$ in $A)$ and $\tau(U)=w$. Since $\left\langle w \oplus w^{*}\right\rangle=\langle 1 \oplus 1\rangle$, the homotopy invariance of $C^{*}(A, U)$ implies that the extension $\sigma: C^{*}(A, U) \rightarrow Q\left(H^{2}\right)$ defined by $\sigma(x)=\pi^{2}(x)$ and $\sigma(U)=$ $w \oplus w^{*}$ is trivial, so there is a $v \in U\left(\pi^{2}(A)^{c}\right)$ such that $v\left(w \oplus w^{*}\right) v^{*}=$ $\pi^{2}(U)$. Set $p=v(1 \oplus 0) v^{*}$, so $p$ commutes with $\pi^{2}\left(C^{*}(A, U)\right)$, hence with $\pi^{2}(\Omega A)$. Furthermore, $p \pi^{2}(U)+1-p=v^{*}(w \oplus 1) v$. Since $\left\langle v^{*}(w \oplus 1) v\right\rangle=\langle w \oplus 1\rangle$, we have $\gamma\left([p]_{0}\right)=\langle w\rangle$.

Theorem 6 yields a poor man's version of the exact sequence in [6] with little effort. Let $\theta$ be an automorphism of $A$ and $C^{*}(A, \theta)$ the crossed product of $A$ by $\theta$. We will consider the latter to be faithfully represented on $H$, missing the compacts, as $C^{*}\left(A, U_{\theta}\right)$, where $U_{\theta}$ is a unitary on $H$ with $U_{\theta} x U_{\theta}^{*}=\theta(x)$ for $x$ in $A$. Write $\Omega \theta$ for the restriction of $\theta \otimes \mathrm{id}$. on $A \otimes C(T)$ to $\Omega A$.

THeOREM 7. Let $A$ be separable and unital, and suppose that $A \otimes C(T)$ and $C^{*}(A, \theta)$ both have the homotopy invariance property. There is an exact sequence

$\operatorname{Ext}^{*}(\Omega A) \stackrel{\alpha_{1}}{\longrightarrow} \operatorname{Ext}^{*}(\Omega A) \stackrel{\delta}{\longrightarrow} \operatorname{Ext}^{*}\left(C^{*}(A, \theta)\right) \stackrel{i_{*}}{\longrightarrow} \operatorname{Ext}^{*}(A) \stackrel{\alpha_{0}}{\longrightarrow} \operatorname{Ext}(A)$, where $\alpha_{1}([\tau])=\left[\tau \circ \Omega \theta^{-1}\right]-[\tau]$ and $\alpha_{0}([\sigma])=\left[\sigma \circ \theta^{-1}\right]-[\sigma]$. 
Proof. We use Theorem 6 to identify $\operatorname{Ext}^{*}(\Omega A)$ with $K_{1}\left(\pi(A)^{c}\right)$; the map $\alpha_{1}: K_{1}\left(\pi(A)^{c}\right) \rightarrow K_{1}\left(\pi(A)^{c}\right)$ is given by

$$
\alpha_{1}(\langle w\rangle)=\left\langle\pi\left(U_{\theta}\right)^{*} w \pi\left(U_{\theta}\right) w^{*}\right\rangle .
$$

To define $\delta$, take $w$ in $K_{1}\left(\pi(A)^{c}\right)$ and define $\tau_{w}: C^{*}(A, \theta) \rightarrow Q\left(H^{2}\right)$ by $\tau_{w}(x)=\pi^{2}(x)$ and $\tau_{w}\left(U_{\theta}\right)=\pi\left(U_{\theta}\right) w \oplus \pi\left(U_{\theta}\right)$. (The universal property of the crossed product enables one to do this, since $\pi\left(U_{\theta}\right) w$ implements $\theta$ on $\pi(A)$.) If $\langle w\rangle=\langle v\rangle$, then $\left[\tau_{w}\right]=\left[\tau_{v}\right]$ because $C^{*}(A, \theta)$ has the homotopy-invariance property, so $\delta: K_{1}\left(\pi(A)^{c}\right) \rightarrow \operatorname{Ext}\left(C^{*}(A, \theta)\right)$ is welldefined by $\delta(\langle w\rangle)=\left[\tau_{w}\right]$. That $\delta$ is additive follows from $\langle w \oplus v\rangle=$ $\langle w v \oplus 1\rangle$; consequently, $\delta$ maps into $\operatorname{Ext}^{*}\left(C^{*}(A, \theta)\right)$. If we set

$$
v=\pi\left(U_{\theta}\right)^{*} w \pi\left(U_{\theta}\right) w^{*},
$$

then $\tau_{v}(\cdot)=(w \oplus 1) \pi^{2}(\cdot)(w \oplus 1)$, so $\delta \alpha_{1}=0$. Suppose $\delta(\langle w\rangle)=0$, i.e., that $\tau_{w}$ is trivial. Then there is a $v$ in $U\left(\pi^{2}(A)^{\circ}\right)$ such that $v^{*}\left(\pi^{2}\left(U_{\theta}\right)\right)(w \oplus 1) v=\pi^{2}\left(U_{\theta}\right)$, which means that $\langle w\rangle=\alpha_{1}(\langle v\rangle)$. We have established exactness at the second term. It is immediate from the definition of $\delta$ that $i_{*} \delta=0$. If $i_{*}([\tau])=0$, then we may assume that $\tau: C^{*}(A, \theta) \rightarrow Q(H)$ satisfies $\tau(x)=\pi(x)$ for $x \in A$. Set $w=$ $\pi\left(U_{\theta}\right)^{*} \tau\left(U_{\theta}\right)$, so $w \pi(x) w^{*}=\pi\left(\theta^{-1} \theta(x)\right)=\pi(x)$, i.e., $w \in \pi(A)^{c}$. We have $[\tau]=\left[\tau_{w}\right]$, since $\tau_{w}$ is the direct sum of $\tau$ with a trivial extension. This establishes exactness at $\operatorname{Ext}^{*}\left(C^{*}(A, \theta)\right)$. If $\tau: C^{*}(A, \theta) \rightarrow Q(H)$ is an extension and $\sigma$ its restriction to $A$ (so $[\sigma]=i_{*}([\tau])$ ), then $\sigma\left(\theta^{-1}(x)\right)=\tau\left(U_{\theta}\right)^{*} \sigma(x) \tau\left(U_{\theta}\right)$ for $x$ in $A$ and so $\sigma_{0} i_{*}=0$. If $\sigma: A \rightarrow Q(H)$ is an extension and $\alpha_{0}([\sigma])=0$, then there is a unitary $v$ in $Q(H)$ such that $v^{*} \sigma(x) v=\sigma\left(\theta^{-1}(x)\right)$ for $x$ in $A$. If we define $\tau: C^{*}(A, \theta) \rightarrow$ $Q\left(H^{2}\right)$ by $\tau(x)=\sigma(x) \oplus \pi(x)(x$ in $A)$ and $\tau\left(U_{\theta}\right)=v \oplus \pi\left(U_{\theta}\right)$, we then have $i_{*}([\tau])=[\sigma]$, so the sequence is exact at the fourth term.

Notice that if $C^{*}\left(\Omega^{n} A, \Omega^{n} \theta\right)$ has the homotopy-invariance property for all $n \geqq 1$ (as would be the case if $C^{*}(A, \theta)$ were g.q.d.), we obtain a one-sided long exact sequence from the sequence in Theorem 7 by appending to it

$$
\cdots \longrightarrow \operatorname{Ext}\left(\Omega^{2} A\right) \longrightarrow \operatorname{Ext}\left(\Omega^{2} A\right) \longrightarrow \operatorname{Ext}\left(C^{*}(\Omega A, \Omega \theta)\right)
$$

on the left. Closing up the sequence in Theorem 7 via maps

$$
\operatorname{Ext}(A) \longrightarrow \operatorname{Ext}\left(\Omega C^{*}(A, \theta)\right) \longrightarrow \operatorname{Ext}(\Omega A)
$$

to obtain the cyclic sequence in [6] appears to be beyond the scope of the methods used here, however.

\section{REFERENCES}

1. W. B. Arveson, Notes on extensions of $C^{*}$-algebras, Duke Math. J., 44 (1977), 329-355. 
2. L. G. Brown, Extensions and the structure of $C^{*}$-algebras, Symp. Math., 20 (1976), 539-566.

3. L. G. Brown, R. G. Douglas and P. A. Fillmore, Extensions of $C^{*}$-algebras, operators with compact self-commutators, and K-homology, Bull. Amer. Math. Soc., 79 (1973), 973-978.

4. E. G. Effros and J. Rosenberg, $C^{*}$-algebras with approximately inner flip, Pacific J. Math., 77 (1978), 417-443.

5. W. L. Paschke and N. Salinas, Matrix algebras over $O_{n}$, Michigan Math. J., 26 (1979), 3-12.

6. M. Pimsner and D. Voiculescu, Exact sequences for K-groups and Ext-groups of certain cross-products $C^{*}$-algebras, J. Operator Theory, 4 (1980), 93-118.

7. M. Pimsner and S. Popa, The Ext-groups of some $C^{*}$-algebras considered by $J$. Cuntz, Rev. Roum. Math. Pures Appl., 23 (1978), 1069-1076.

8. M. Pimsner, S. Popa, and D. Voiculescu, Homogeneous $C^{*}$-extensions of $C(X) \otimes$ $K(H)$, part $I$, J. Operator Theory, 1 (1979), 55-108.

9. S. Popa and M. A. Rieffel, The Ext groups of the $C^{*}$-algebras associated with irrational rotations, J. Operator Theory, 3 (1980), 271-274.

10. N. Salinas, Homotopy invariance of $\operatorname{Ext}(A)$, Duke Math. J., 44 (1977), 777-793.

11. J. L. Taylor, Banach algebras and topology, in "Algebras in Analysis", Academic Press (1975), 118-186.

12. D. Voiculescu, A non-commutative Weyl-von Neumann theorem, Rev. Roum. Math. Pures Appl., 21 (1976), 97-113.

Received June 2, 1980. Research for this paper partially supported by NSF Grant MCS 77-01850.

UNIVERSITY OF KANSAS

LAWRENCE, KS 66045 


\section{PACIFIC JOURNAL OF MATHEMATICS}

\section{EDITORS}

DONALD BABBITT (Managing Editor)

University of California

Los Angeles, CA 90024

HUGo RossI

University of Utah

Salt Lake City, UT 84112

C. C. MOORE and ANDREW OGG

University of California

Berkeley, CA 94720
J. DugundjI

Department of Mathematics

University of Southern California

Los Angeles, CA 90007

R. FinN and J. Milgram

Stanford University

Stanford, CA 94305

\section{ASSOCIATE EDITORS}
R. ARENS
E. F. BECKENBACH
B. H. NeumanN
F. WOLF
K. YOSHIDA

\section{SUPPORTING INSTITUTIONS}

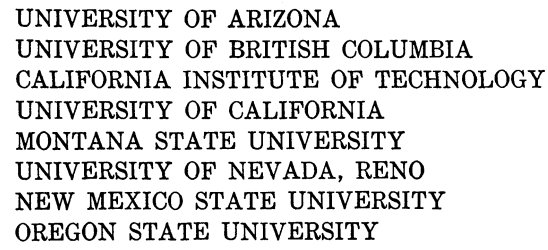

UNIVERSITY OF ARIZONA

UNIVERSITY OF BRITISH COLUMBIA

CALIFORNIA INSTITUTE OF TECHNOLOGY

UNIVERSITY OF CALIFORNIA

MONTANA STATE UNIVERSITY

UNIVERSITY OF NEVADA, RENO

NEW MEXICO STATE UNIVERSITY

OREGON STATE UNIVERSITY

\author{
UNIVERSITY OF OREGON \\ UNIVERSITY OF SOUTHERN CALIFORNIA \\ STANFORD UNIVERSITY \\ UNIVERSITY OF HAWAII \\ UNIVERSITY OF TOKYO \\ UNIVERSITY OF UTAH \\ WASHINGTON STATE UNIVERSITY \\ UNIVERSITY OF WASHINGTON
}

The Supporting Institutions listed above contribute to the cost of publication of this Journal, but they are not owners or publishers and have no responsibility for its content or policies.

Mathematical papers intended for publication in the Pacific Journal of Mathematics should be in typed form or offset-reproduced, (not dittoed), double spaced with large margins. Please do not use built up fractions in the text of the manuscript. However, you may use them in the displayed equations. Underline Greek letters in red, German in green, and script in blue. The first paragraph or two must be capable of being used separately as a synopsis of the entire paper. Please propose a heading for the odd numbered pages of less than 35 characters. Manuscripts, in triplicate, may be sent to any one of the editors. Please classify according to the scheme of Math. Reviews, Index to Vol. 39. Supply name and address of author to whom proofs should be sent. All other communications should be addressed to the managing editor, or Elaine Barth, University of California, Los Angeles, California, 90024.

50 reprints to each author are provided free for each article, only if page charges have been substantially paid. Additional copies may be obtained at cost in multiples of 50 .

The Pacific Journal of Mathematics is issued monthly as of January 1966. Regular subscription rate: $\$ 102.00$ a year (6 Vols., 12 issues). Special rate: $\$ 51.00$ a year to individual members of supporting institutions.

Subscriptions, orders for numbers issued in the last three calendar years, and changes of address shoud be sent to Pacific Journal of Mathematics, P.O. Box 969, Carmel Valley, CA 93924, U.S.A. Old back numbers obtainable from Kraus Per!odicals Co., Route 100, Millwood, NY 10546.

PUBLISHED BY PACIFIC JOURNAL OF MATHEMATICS, A NON-PROFIT CORPORATION

Printed at Kokusai Bunken Insatsusha (International Academic Printing Co., Ltd.). 8-8, 3-chome, Takadanobaba, Shinjuku-ku, Tokyo 160, Japan. 


\section{Pacific Journal of Mathematics}

\section{Vol. 95, No. $2 \quad$ October, 1981}

George E. Andrews, The Rogers-Ramanujan reciprocal and Minc's

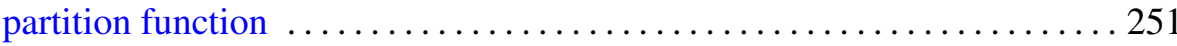

Allan Calder, William H. Julian, Ray Mines, III and Fred Richman,

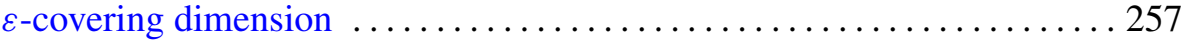

Thomas Curtis Craven and George Leslie Csordas, An inequality for the distribution of zeros of polynomials and entire functions $\ldots \ldots \ldots \ldots 263$

Thomas Jones Enright and R. Parthasarathy, The transfer of invariant

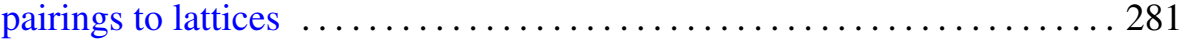

Allen Roy Freedman and John Joseph Sember, Densities and

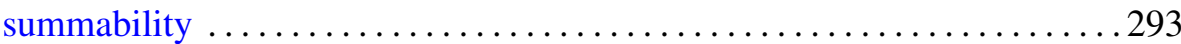

Robert Heller and Francis Aubra Roach, A generalization of a classical necessary condition for convergence of continued fractions . . . . . . 307

Peter Wilcox Jones, Ratios of interpolating Blaschke products ........... 311

V. J. Joseph, Smooth actions of the circle group on exotic spheres ........ 323

Mohd Saeed Khan, Common fixed point theorems for multivalued

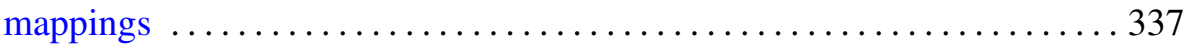

Samuel James Lomonaco, Jr., The homotopy groups of knots. I. How to

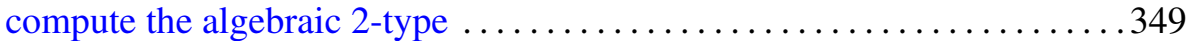

Louis Magnin, Some remarks about $C^{\infty}$ vectors in representations of connected locally compact groups ............................ 391

Mark Mandelker, Located sets on the line . . . . . . . . . . . . . . . . . 401

Murray Angus Marshall and Joseph Lewis Yucas, Linked quaternionic mappings and their associated Witt rings $\ldots \ldots \ldots \ldots \ldots \ldots \ldots \ldots . \ldots \ldots 11$

William Lindall Paschke, $K$-theory for commutants in the Calkin algebra

W. J. Phillips, On the relation $P Q-Q P=-i I$ 435

Ellen Elizabeth Reed, A class of Wallman-type extension. 443

Sungwoo Suh, The space of real parts of algebras of Fourier transforms 461 Antonius Johannes Van Haagen, Finite signed measures on function

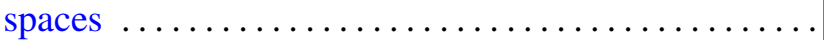

Richard Hawks Warren, Identification spaces and unique uniformity 